\title{
Thermoelectrical characterization of piezoelectric diaphragms: towards a better understanding of ferroelectrics for future memory applications.
}

\author{
G. Vinuesa, P. Marín, Ó. G. Ossorio, B. Sahelices, H. García, H. Castán and S. Dueñas. \\ Department of Electronics, University of Valladolid, Valladolid 47011, SPAIN
}

\begin{abstract}
This work deals with the thermoelectric characterization of commercial lead zirconate titanate (PZT) based piezoelectric diaphragms. An in-depth analysis of the piezo- and ferroelectric behavior of the samples was carried out by measuring currentvoltage curves and polarization hysteresis cycles in a wide temperature range. We demonstrate that, as the temperature decreases, higher electric fields are needed to completely polarize the sample. Furthermore, I-V measurements, polarization hysteresis loops and coercive fields allow us to confirm that the samples grain sizes directly correlate with the observed characteristics. From impedance measurements, resonance frequency values, capacitance and permittivity were determined at temperatures ranging from 100 to $320 \mathrm{~K}$. It is shown that impedance maxima shift towards greater frequency values when decreasing the temperature. That is mainly attributed to the appearance of an internal stress that generates larger stiffness in the ceramic. Finally, an electroacoustic characterization was made by measuring the diaphragms characteristics due to sound waves in the human-hearing frequency range.
\end{abstract}

\section{Introduction}

Piezoelectric diaphragms, more commonly known as buzzers, are electroacoustic components based on the inverse piezoelectric effect (1). This means that, when an electric field is applied in the polarization direction of the piezoelectric, a mechanical deformation in the opposite direction is produced, resulting a human-audible sound in the case of buzzers (2). Because of this, their most common application so far has been the integration of alarm systems. However, their range of application has increased over the years, and recent studies propose piezoelectric diaphragms as possible sensors for Structural Health Monitoring (SHM) (3-5). Silva de Freitas et al. $(6,7)$ studied lead zirconate titanate (PZT) based buzzers to determine their application in SHM as damage detectors, studying both their temperature stability and their temperature-frequency dependence from 0 to $70^{\circ} \mathrm{C}$.

Several studies have been carried out on $\mathrm{Pb}\left(\mathrm{Mg}_{1 / 3} \mathrm{Nb}_{2 / 3}\right) \mathrm{O}_{3}-\mathrm{PbTiO}_{3}$ piezoelectric crystals (8) and lead-free ceramics such as $0.965\left(\mathrm{~K}_{0.45} \mathrm{Na}_{0.55}\right)\left(\mathrm{Nb}_{0.96} \mathrm{Sb}_{0.04}\right) \mathrm{O}_{3}$ $0.0375 \mathrm{Bi}_{0.5} \mathrm{Na}_{0.5} \mathrm{Zr}_{0.85} \mathrm{Hf}_{0.15} \mathrm{O}_{3}(9),\left(\mathrm{K}_{0.5-x} \mathrm{Li}_{x}\right) \mathrm{Na}_{0.5}\left(\mathrm{Nb}_{1-y} \mathrm{Sb}_{y}\right) \mathrm{O}_{3}$ (10) and BaTiO3 (11) in order to study their ferroelectric behavior and properties (coercive field, impedance, hysteresis loop) and their dependence with frequency, as well as to further understand the dependencies of the dielectric permittivity with these magnitudes (12). Likewise, ferroelectric materials are interesting for many technological purposes $(13,14)$ and are suitable components to fabricate non-volatile memories (FeRAM) $(15-20)$, as a possible 
replacement of gate oxides in dynamic random-access memories (DRAMs) (20), or as the gate stack of ferroelectric field-effect transistors (FeFET) $(20,21)$.

Since every ferroelectric is in fact piezoelectric (and pyroelectric (22)), piezoelectric ceramics are good challengers to be investigated as ferroelectric materials. In the search of materials with high charge storage densities to be used in the memory field, metal oxide perovskites such as lead zirconate titanate (PZT) is of particular interest due to their low leakage currents. Besides, ferroelectric PZT shows high remanent polarization, high permittivity, and thermal stability with low coercive field (23). These properties make this material promising for high-speed and low-voltage non-volatile memories (24). Additionally, PZT presents a high electromechanical coupling coefficient, which means that they can be easily poled and feature a very high Curie Temperature. That allows PPZT to be fully operational across a wide temperature range (25).

In this work we have carried out an in-depth study of ferroelectric behavior, measuring the change in ferro- and piezoelectric properties with temperature (and frequency in the case of piezoelectricity). We focused our study at low temperatures, where previous research is scarce.

\section{Experimental Setup}

The devices under investigation were piezoelectric diaphragms (buzzers) manufactured by MuRata Manufacturing Co., Ltd.. Measurements were carried for the models 7BB-129 and 7BB-20-6. The active element in these buzzers is not given by the manufacturer, but as it can be deduced from the results of Silva de Freitas et al. $(6,7)$. The main compound used is PZT (although, as it will be further addressed in this paper, it is not the only one), very common for its good behavior as both ferro- and piezoelectric. For the sake of simplification, we will refer to these models as SD (Small Device) for the 7BB-12-9 model with $9 \mathrm{~mm}$ diameter and $0.022 \mathrm{~mm}$ thickness, and LD (Large Device) for the 7BB-20-6 model with $12 \mathrm{~mm}$ diameter and $0.042 \mathrm{~mm}$ thickness. Dimensions and properties of the buzzers can be seen in Table I.

TABLE I. Dimensions and properties of the 7BB-12-9 and 7BB-20-6 piezoelectric diaphragms manufactured by MuRata.

\begin{tabular}{ccc}
\hline Feature & 7BB-12-9 (SD) & 7BB-20-6 (LD) \\
\hline Resonance $(\mathrm{kHz})$ & 9 & 6.3 \\
Resonant freq. tolerance $(\mathrm{kHz})$ & \pm 1 & \pm 0.6 \\
Capacitance $(\mathrm{nF})$ & 8 & 10 \\
Capacitance tolerance & $\pm 30 \%$ & \pm 30 \\
Plate Diameter $(\mathrm{mm})$ & $12 \pm 0.2$ & $20 \pm 0.2$ \\
Active Element Diameter(mm) & $9 \pm 0.6$ & $14 \pm 0.6$ \\
Plate Thickness $(\mathrm{mm})$ & $0.10 \pm 0.05$ & $0.20 \pm 0.05$ \\
Active Element Thickness $(\mathrm{mm})$ & $0.22 \pm 0.10$ & $0.42 \pm 0.10$ \\
Plate Material & Brass & Brass \\
\hline
\end{tabular}

Electrical characterization of the buzzers was carried out by means of an Agilent B2987A electrometer. Current-Voltage curves were obtained by applying a positive 
voltage ramp until the buzzer was completely polarized. Afterwards, a negative voltage ramp was applied on the opposite direction, thus performing a double sweep which delivered a bi-valued function. The hysteresis loop, presented as a charge-voltage curve, was acquired by integrating the current values measured when applying voltage to the piezoelectric diaphragm.

To analyze the buzzers impedance response, we used a circuit consisting in a voltage divider as shown in Fig. 1. An Agilent 33250A wave generator was used to apply sinusoidal voltage waveforms with frequencies ranging from $5 \mathrm{kHz}$ to $20 \mathrm{kHz}$. It is easy to calculate the divider transfer function as:

$$
H(\omega)=\frac{V_{\text {in }}}{V_{\text {out }}}=1+Z / R
$$

where $\mathrm{R}$ is the internal impedance of the oscilloscope set at $50 \Omega$ and $\mathrm{Z}$ is the sample impedance. The real, $X$, and imaginary, $Y$, parts of the impedance, $Z$, can be derived from the module, $M$, and phase, $\varphi$, of $H(\omega)$.

$$
M \cos (\varphi)=1+X / R \quad ; M \sin (\varphi)=Y / R
$$

The digital oscilloscope captures $V_{\text {in }}$ and $V_{\text {out }}$ waveforms and, subsequently, $\mathrm{M}$ and $\varphi$ are calculated. Finally, $\mathrm{X}, \mathrm{Y}$, and thus, $\mathrm{Z}$ are obtained as follows:

$$
\begin{aligned}
& X=R(M \cos (\varphi)-1) \\
& Y=R M \sin (\varphi) \\
& Z=\sqrt{X^{2}+Y^{2}}
\end{aligned}
$$

These measurements were carried out at temperatures from $100 \mathrm{~K}$ to $320 \mathrm{~K}$ by placing the samples in a liquid nitrogen cryostat.

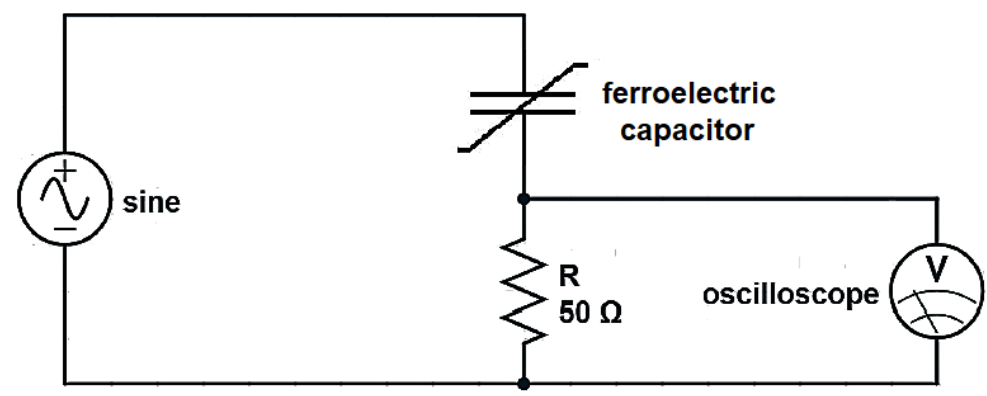

Figure 1. Schematic representation of the Voltage Divider circuit. 


\section{Results and Discussion}

$\underline{\text { Polarization hysteresis }}$

To obtain the Small Device polarization curve for both positive and negative voltages, we applied a voltage ramp from 0 to $150 \mathrm{~V}$ which guaranteed the visualization of the entire curve at $300 \mathrm{~K}$. The I-V curve and Hysteresis loop (Fig.2) confirmed that the ceramic used in the buzzers showed an excellent ferroelectric behavior.
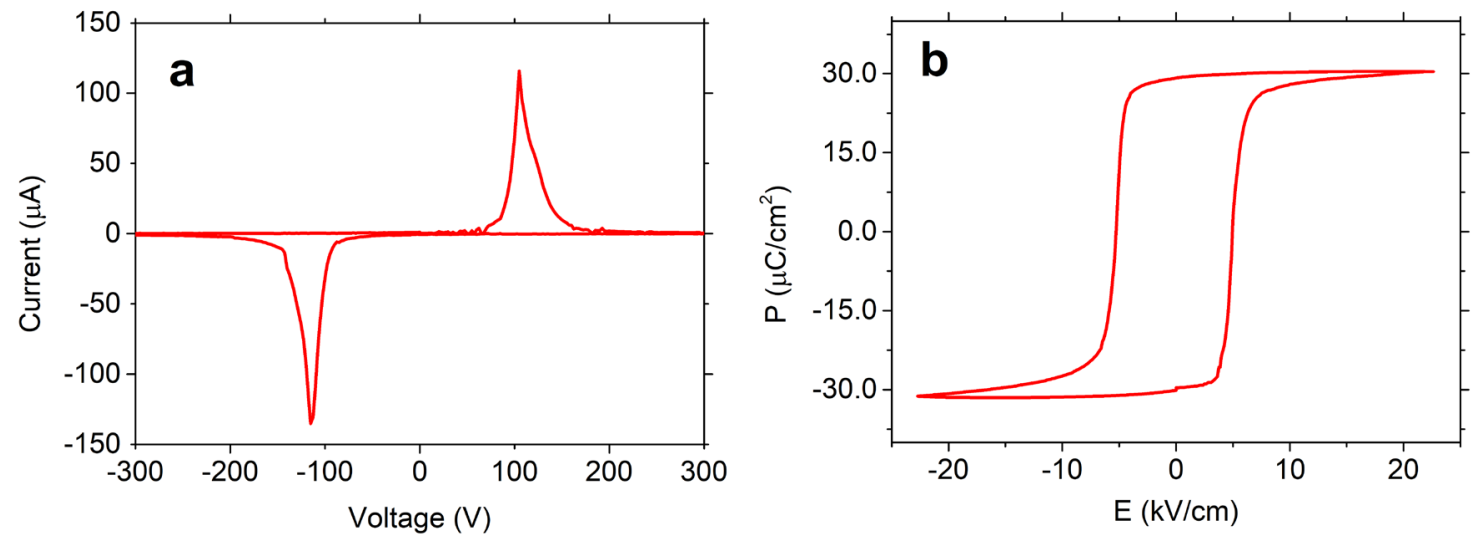

Figure 2: I-V curve (a) and polarization hysteresis loop (b) of the small device at $300 \mathrm{~K}$.

Subsequently, the sample was introduced into the cryostat and we performed the same measurements at temperatures from 100 to $320 \mathrm{~K}$ with steps of $10 \mathrm{~K}$. As we will further address in this paper, the acquisition of the curves for all thermal conditions required an

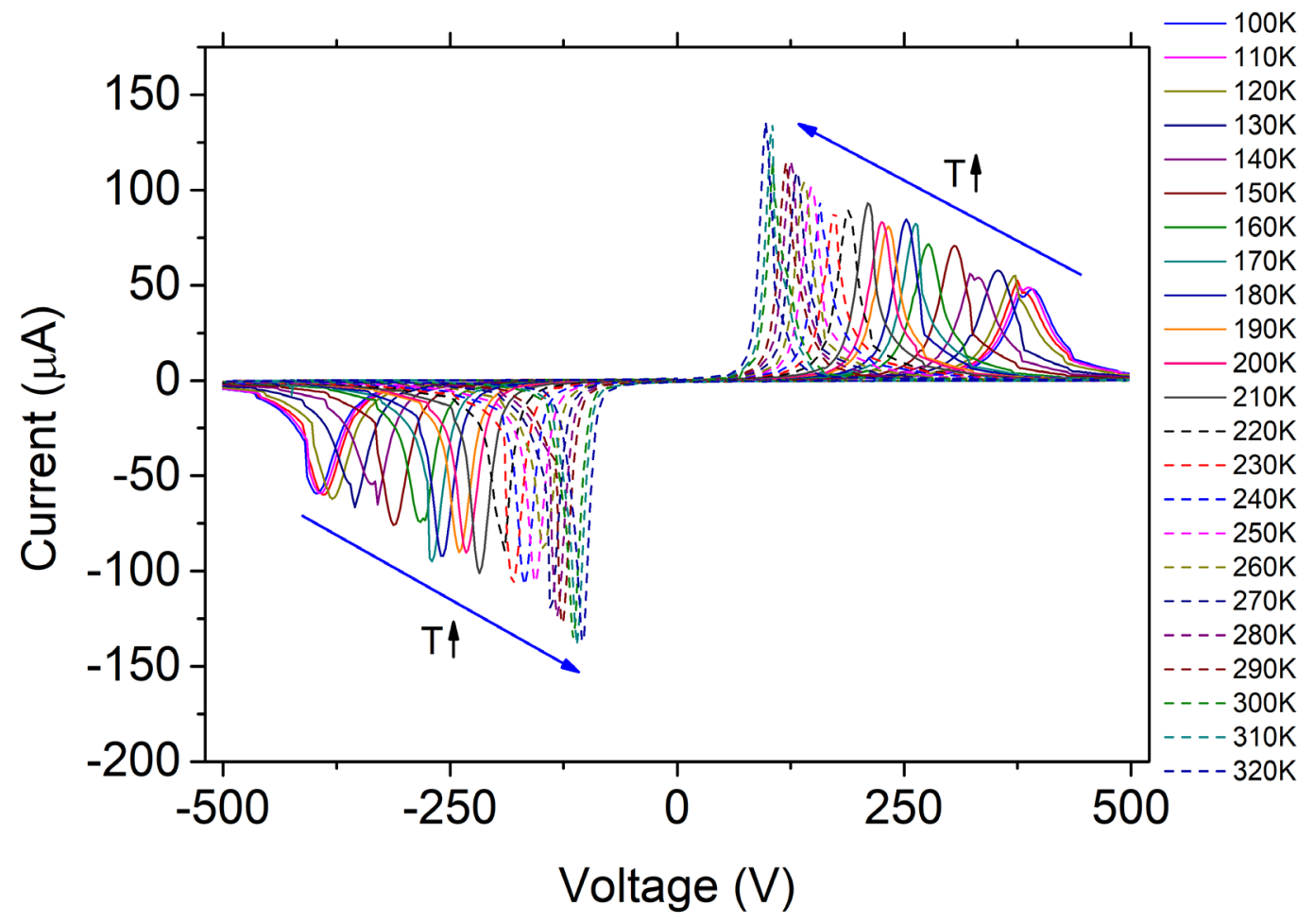

Figure 3: SD I-V polarization curves at different temperatures in the range 100-320 K 
increase in the maximum value of the voltage ramp up to $500 \mathrm{~V}$, with no reported impact in the integrity of the sample.

In Fig. 3, we observe that the lower the temperature of the sample, the higher the voltage needed in order to fully polarize the ceramic. Moreover, the current maximum obtained by the polarization process dropped smoothly as temperature decreased. This suggested us that the lower the temperature of the sample, the weaker the activation of the inverse piezoelectric effect. Thus, a higher electric field is needed to polarize the sample. This behavior can be explained because ferroelectric polarization is due to ferroelectric domain wall motion, a thermally activated process (26). Consequently, as temperature decreases, domain reversal becomes gradually tougher. Therefore, the electric fields needed to polarize the sample become higher. In addition, the decrease in polarization current could be attributed to the inability to reverse all the domains at such low temperatures. The fact that domain walls lose mobility and cannot be reversed in their entirety as the temperature lowers explains the increase of the coercive field as temperature decreases $(27,28)$. Fig. 4 presents a 3D plot (Q-V-T) of the hysteresis loop where the rise of the coercive field with temperature is noticeable (since $\mathrm{E}$ and $\mathrm{V}$ are proportional to each other). The gap between both the first and the last charge values in the hysteresis loops is the result of different polarization states at the beginning and the end of the experiment.

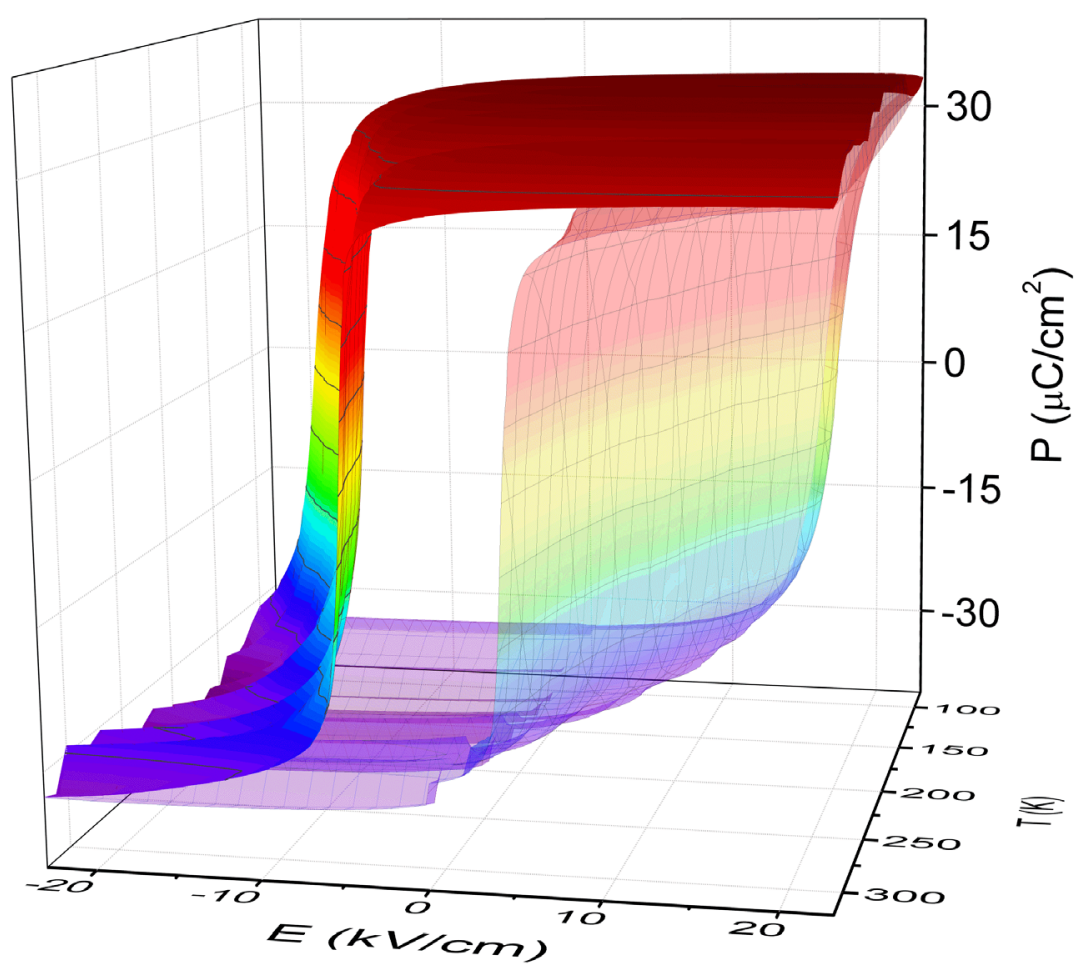

Figure 4. Variation of the small device polarization hysteresis loop with temperature. Warmer (cooler) colors indicate higher (lower) charge values.

\section{$\underline{\text { Size dependence }}$}

To measure possible size dependences, the same experiments described above were carried for a new diaphragm model 7BB-20-6 (LD) which differs from the previous one in 
its element size ( $14 \mathrm{~mm}$ ceramic diameter vs. the SD's $9 \mathrm{~mm}$ diameter). The temperature sweep (Fig. 5) showed that the behavior of the current peaks followed the same pattern observed in the first sample (Fig. 3). In Fig. 6 we compare current densities and polarization loops of both samples. Fig. 6(a) shows that, at $300 \mathrm{~K}$, the peak corresponding to the current density of the LD model almost doubles the height of that of the SD. Moreover, Fig. 6(b) presents a great difference between the polarization hysteresis of the buzzers. The smaller one shows higher coercive field values and much bigger electric fields to be completely polarized and achieve saturation values. These differences may be explained by domain wall mobility and type, determined by the different thicknesses of our samples.

Ferroelectric materials present two types of domain walls: $180^{\circ}$ and non- $180^{\circ}$ domain walls. Usually, when studying thin films, only the $180^{\circ}$ domain wall contribution is important. However, both devices studied present large thickness (far above $2 \mu \mathrm{m}$ ), making the contribution of non- $180^{\circ}$ domain wall motion non-negligible (26). As the LD is not only larger, but thicker than the SD, finer grains are expected for the latter (28). Consequently, lesser $180^{\circ}$ domain wall density and greater $180^{\circ}$ domain wall pinning (reduced mobility) is expected for the smaller buzzer. Moreover, it is possible that non$180^{\circ}$ domain wall contribution is greater in thicker films with larger grains (26). These mechanisms would explain why the polarization current densities shown in Fig. 6a are lower and wider for the smaller device, as well as the greater coercive field measured and electric field values needed seen for the SD (Fig. 6b).

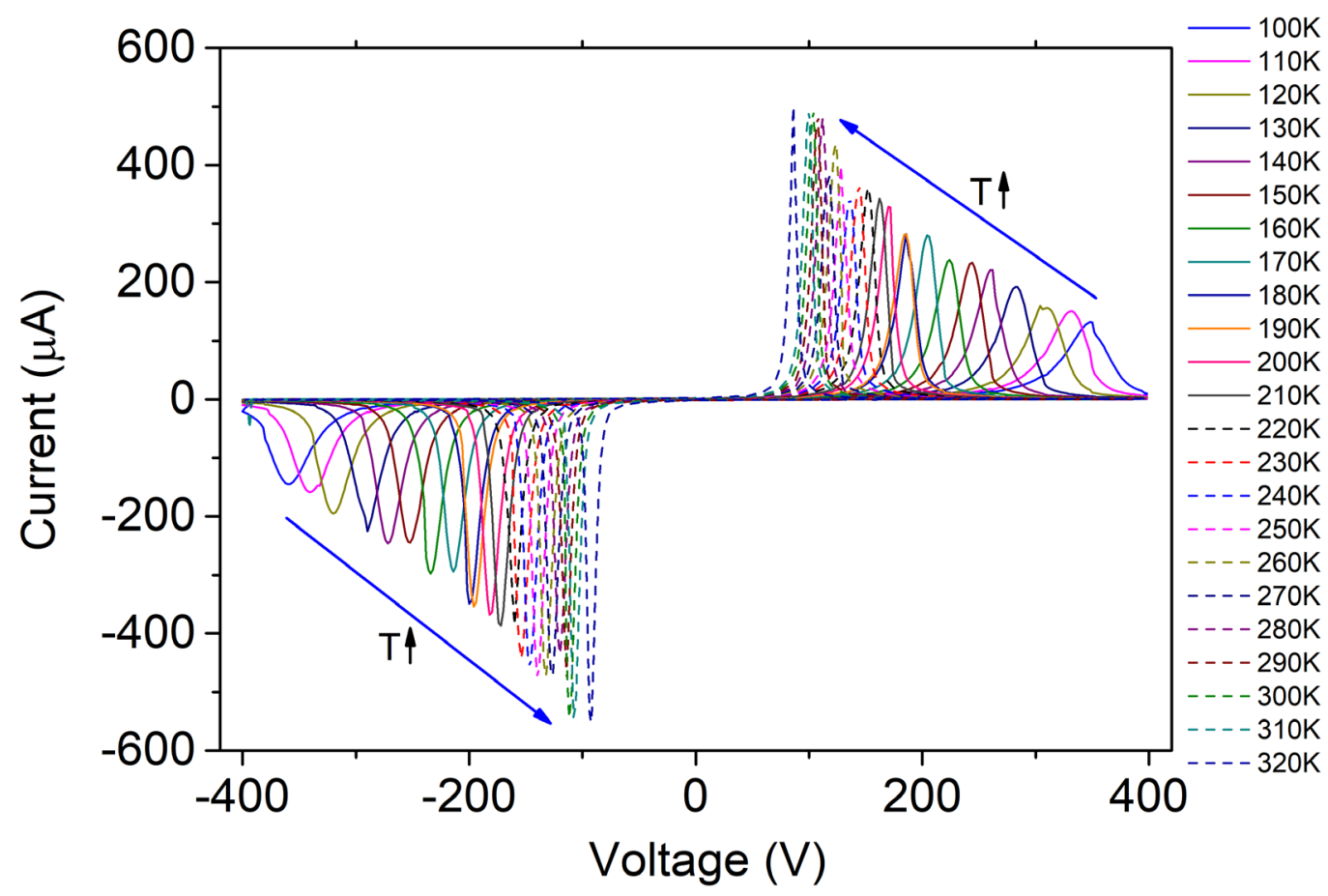

Figure 5: I-V polarization curves of the large device at different tempeatures in the range $100-320 \mathrm{~K}$. 

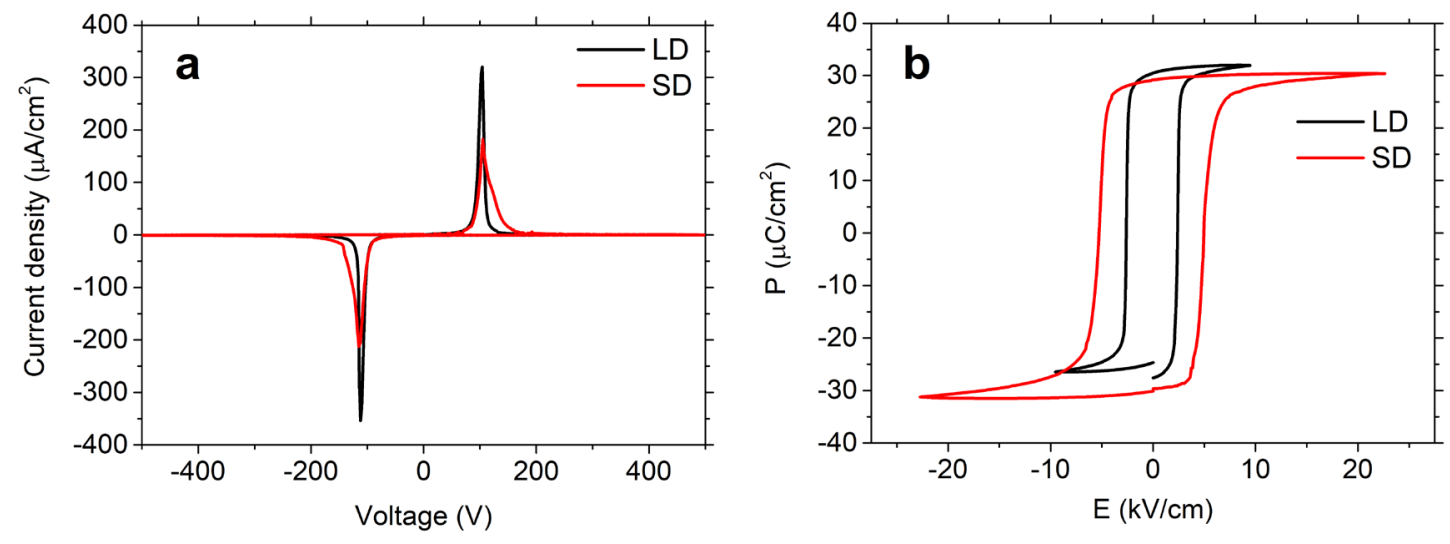

Figure 6: Current density versus voltage (a) hysteresis loops (b) of the small (red) and large (black) devices at $300 \mathrm{~K}$.

Furthermore, we measured the relationship between the magnitude of the coercive field and temperature corresponding to both devices (Fig. 7). Similar behavior was previously found by Xu et al. (26) and Meng et al. (27). The rise of the coercive field as the temperature decreases is because domain wall motion is thermally activated. Hence, as the temperature lowers, the inability to reverse all domain walls combines with the reduced mobility of the reversible ones. Some of them result blocked, i.e., unable to be reoriented (31). Therefore, higher electric field values must be applied, and lower poling currents are measured. Even though this behavior is common for both samples, we cannot ignore that, in fact, there is a dependence in the poling processes of the samples regarding their size. It has been reported that the size of the sample affects the poling process $(26,27)$ as well as the coercive field $(28,29)$. In our case, the smaller the diaphragm, the lower mobility of the polarization

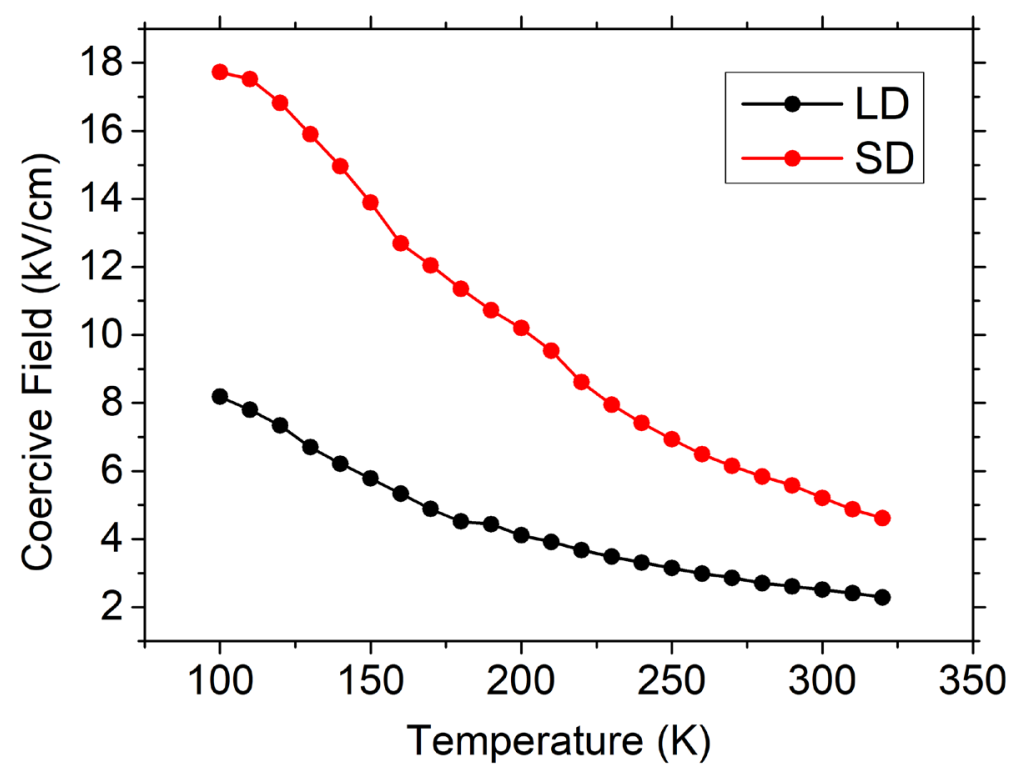

Figure 7: Coercive Field of the small (red) and large (black) piezoelectric diaphragms in the $100-320 \mathrm{~K}$ temperature range. 
domain walls, causing the coercive field to rise as seen in Fig. 7. These results indicate that, domain reversal becomes more difficult as the sample is thinner. As we have previously explained, less thickness implies less $180^{\circ}$ domain wall mobility, as well as finer grains (which reduce the non- $180^{\circ}$ domain reversal).

The phenomenon of polarization backswitching causes the difference between the remanent polarization and the saturation polarization (30). This mechanism opposes the nucleation and mobility of domain walls (31-35), acting as a driving force against the alignment of the polarization domains. It is interesting to analyze this magnitude with a view to the ferroelectrics' future memory applications, as it works against their non-volatile effect. Fig. 8 shows the Arrhenius plot of polarization backswitching of the two different size buzzers. It agrees the fact that this phenomenon is more pronounced at higher temperatures as found by Wen et al. (36). Moreover, our data expand the studied range of this phenomenon and confirms that it follows an Arrhenius law at very low temperatures (36).

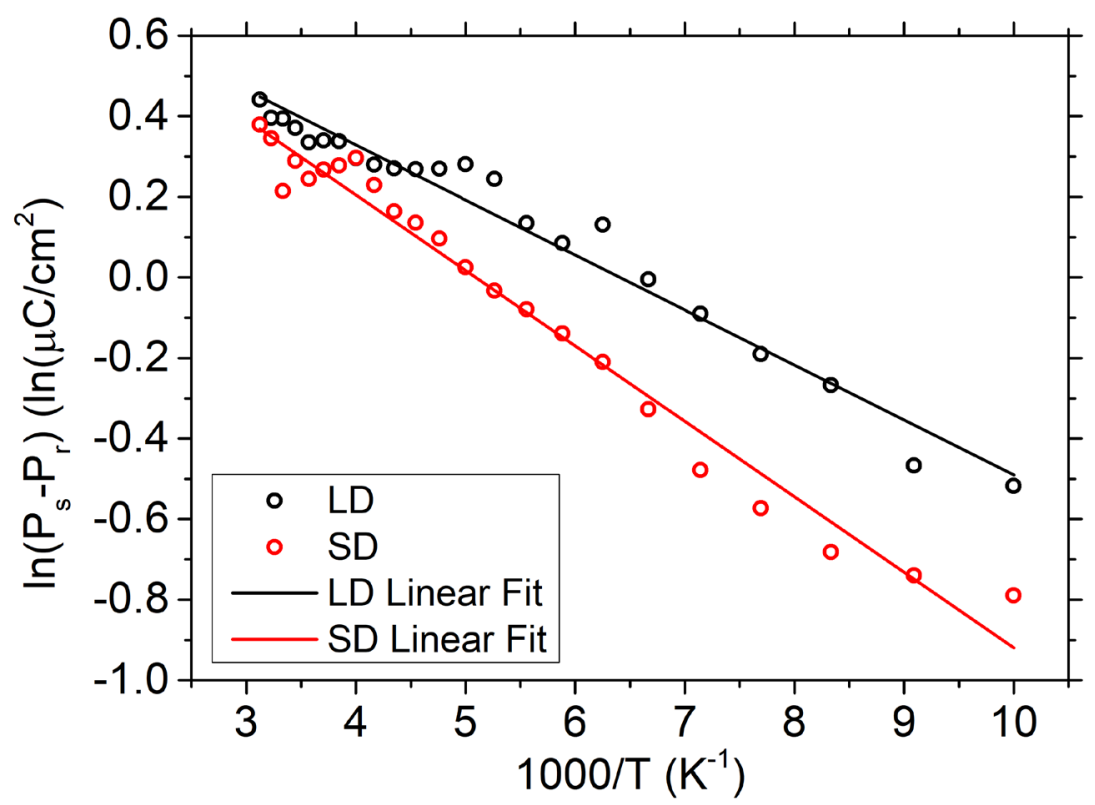

Figure 8: Arrhenius plot and linear fit of the small (red) and large (black) devices polarization backswitching evolution with temperature.

Knowing that the relationship between $P_{S}-P_{r}$ and T follows:

$$
P_{S}-P_{r}=P_{0} \exp \left(-\frac{E_{A}}{k_{B} \cdot T}\right)
$$

where $E_{A}$ is the activation energy, and $P_{0}$ is a constant. We can estimate the activation energy values of domain switching by the slopes obtained when plotting $\ln \left(P_{S}-P_{r}\right)$ vs. $-1 / k_{B} T$. The energies obtained are $0.016 \mathrm{eV}$ and $0.012 \mathrm{eV}$ for the small and large devices, respectively. 
The fact that the thicker buzzer presents the smaller $E_{A}$ value supports our hypothesis that its grains are larger, and thus, its domain wall mobility (both $180^{\circ}$ and non$180^{\circ}$ ) is greater. Furthermore, the low activation energy values may indicate that the samples present a typical relaxor ferroelectric behavior (37).

\section{Impedance analysis}

In this section we present the impedance measurements, which were only carried out for the SD. Fig. 9 plots the electrical impedance (impedance from now on) at $300 \mathrm{~K}$ of the small device. We identified several resonances at $6.4 \mathrm{kHz}, 11.6 \mathrm{kHz}, 15.3 \mathrm{kHz}$ and $17 \mathrm{kHz}$ besides from the one given by the manufacturer at $8.3 \mathrm{kHz}$ (which is in fact the global impedance maximum (anti-resonance)); in spite of this findings, we believe that $11.6 \mathrm{kHz}$ and $17 \mathrm{kHz}$ may be harmonic frequencies of the $6.4 \mathrm{kHz}$ and $8.3 \mathrm{kHz}$ resonances. However, the same cannot be said for the $15.3 \mathrm{kHz}$ one.

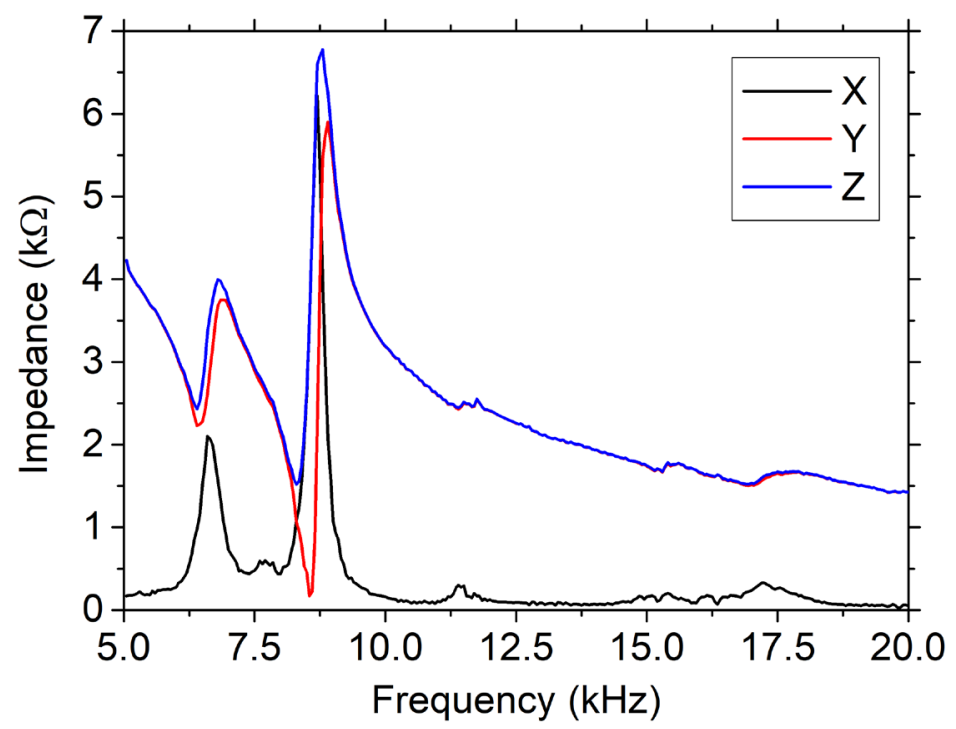

Figure 9: Magnitude (Z), imaginary (Y) and real (X) parts of electrical impedance as a function of the frequency measured for the small device at room temperature and at zero electric field.

To carry out a more careful analysis of these resonances while ensuring the validity of the results, we performed a sound level analysis. At room temperature, a sound level meter was placed in front of our piezoelectric diaphragm, and the frequency sweep was carried as previously described. Results are plotted in Fig. 10. The sound pressure maxima fit well with the resonances observed in Fig. 9. However, the response between 15.3 and $17 \mathrm{kHz}$ is much significant in comparison with the impedance ones, and the $11.6 \mathrm{kHz}$ peak is missing. A better analysis is obtained by comparing the sound pressure spectra with the phase displacement between the input and output electric waveforms. In Fig. 11, we plot the phase shift and the capacitance as a function of frequency. This plot clearly confirms the results obtained in Fig. 10. Moreover, this shows that the buzzer has in fact fine electroacoustic properties in the human audible frequency range, which would make it suitable for alarms or SHM applications. 


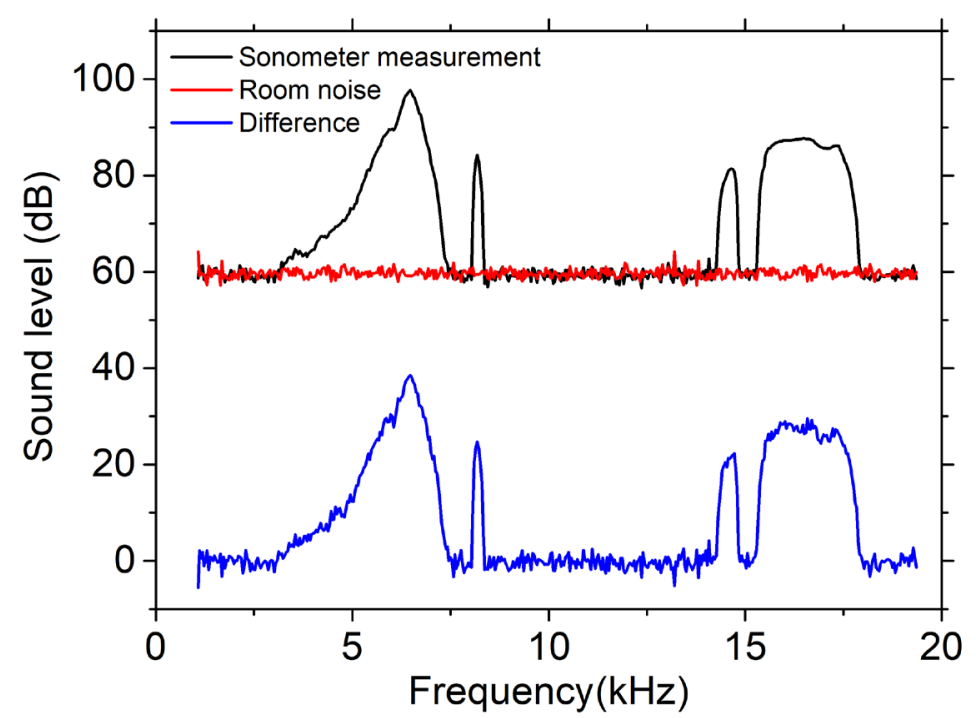

Figure 10: Sound pressure levels at low frequencies for the small device.

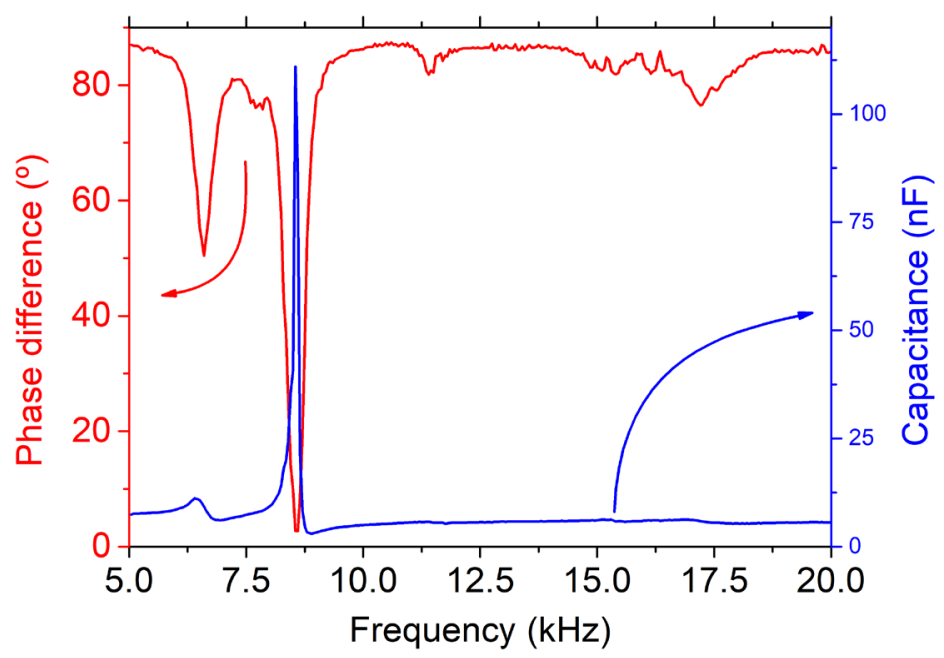

Figure 11: Phase difference (red) and capacitance (blue) at low frequencies for the small device

Subsequently, the SD was placed inside the cryostat to carry out the electroacoustic characterization as a function of temperature. The results provided by Fig. 12 indicate that the anti-resonance peaks get sharper and higher continuously as temperature decreases and, interestingly, the $6.4 \mathrm{kHz}$ peak surpasses that of $8.3 \mathrm{kHz}$ in height. The flattening of the sharp impedance peaks with growing temperature recorded in Fig. 13 has been observed in a previous work by G. Park et al. (38). Following their reasoning, it was attributed to a decrease in the dynamic interaction between the PZT patch and the two electrodes of the buzzer (38) 

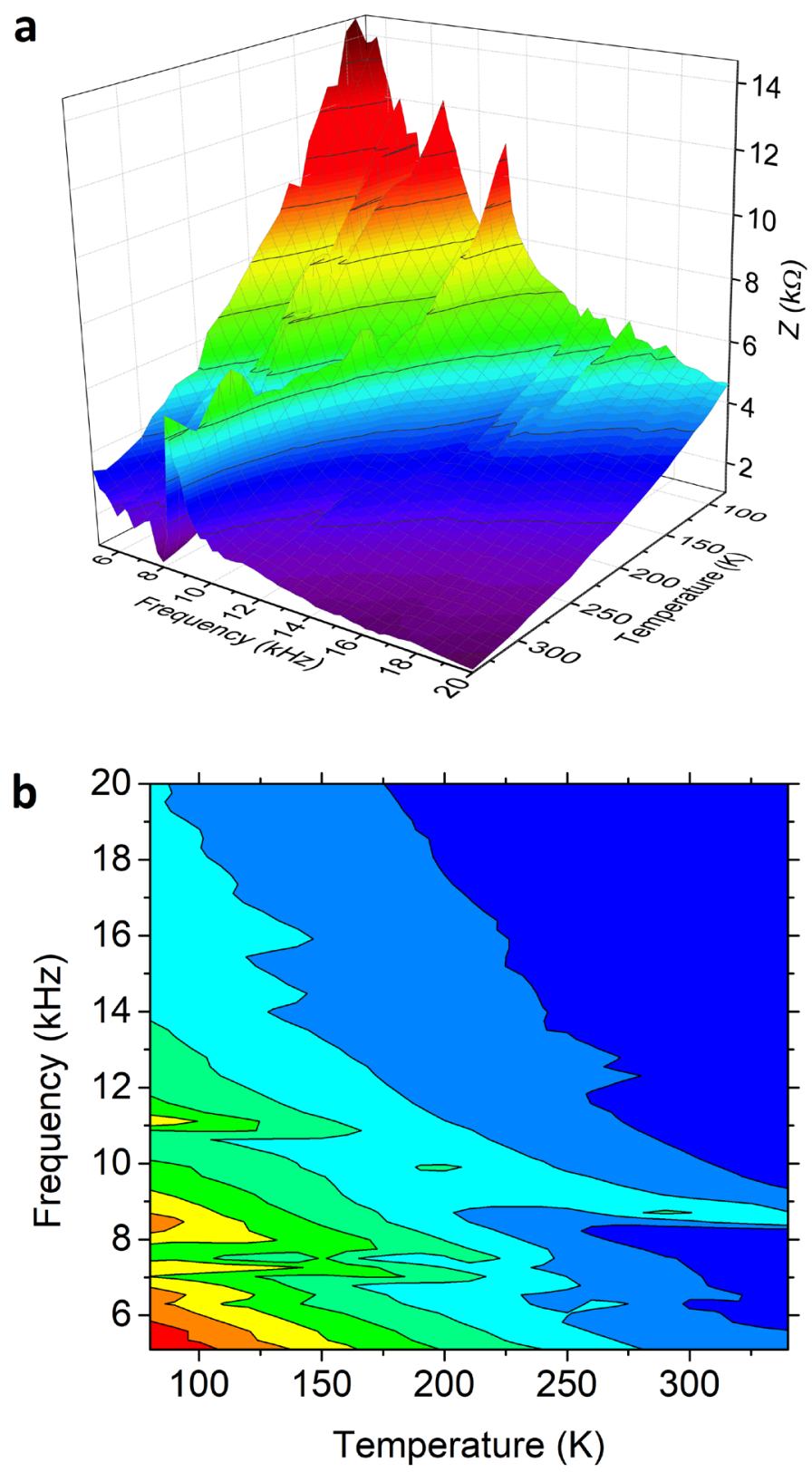

Figure 12:3D surface and Contour plots of the impedance as a function of frequency and temperature. The shift towards higher frequencies at lower temperatures can be clearly noticed. Warmer (cooler) colors indicate higher (lower) Z values.

We claim that the fact that the impedance values rise as temperature decreases is a result of the inversely squared proportional relationship between the electrical impedance and the piezoelectric coefficients (Liang et al. (39)). Silva de Freitas et al. (7) proved that the inverse piezoelectric effect is dominated by these piezoelectric coefficients which affect the impedance value (39), and depend both on frequency $(40,41)$ and temperature $(42)$. Figure 12 clearly shows that the anti-resonances shift towards higher frequencies as the temperature decreases. Similar behavior has been previously observed by Lim et al. (43) by applying tensile stress to PZT patches. This leads us to believe that the temperature 
change experimented by our buzzer was in fact a source of internal stress inducing a stiffening effect to the piezoelectric ceramic. Consequently, the piezoelectric coefficients reduced their value, resulting in higher impedance values (39).

Additionally, we have obtained the dielectric permittivity, $\varepsilon$, as a function of the temperature and frequency. First, we computed the values for capacitance of the PZT ceramic, shown in Fig. 11 at $300 \mathrm{~K}$ and in Fig. 12 for the entire temperature range, using the following expression:

$$
C=1 /(\omega Y)
$$

where $\omega$ is the angular frequency $(\omega=2 \pi f)$. The relative dielectric permittivity is given by:

$$
\varepsilon_{r}=(C t) /\left(A \varepsilon_{0}\right)=\varepsilon / \varepsilon_{0}
$$

where $A$ is the electrode area, $t$ is the ceramic layer thickness, and $\varepsilon_{0}$ is the vacuum permittivity. We focus on the $10-20 \mathrm{kHz}$ range where the capacitance presents an almostconstant value (see Fig. 11). As there was no observed electric permittivity dependence with frequency in this range, Fig. 13 shows $\varepsilon_{r}$ at $20 \mathrm{kHz}$. It can be seen that the relative dielectric constant greatly increases with temperature from 672 at $80 \mathrm{~K}$ to 2841 at $340 \mathrm{~K}$. This is consistent with the results previously presented by Xu et al. (26), Meng et al. (27) and Sabat et al. (44).

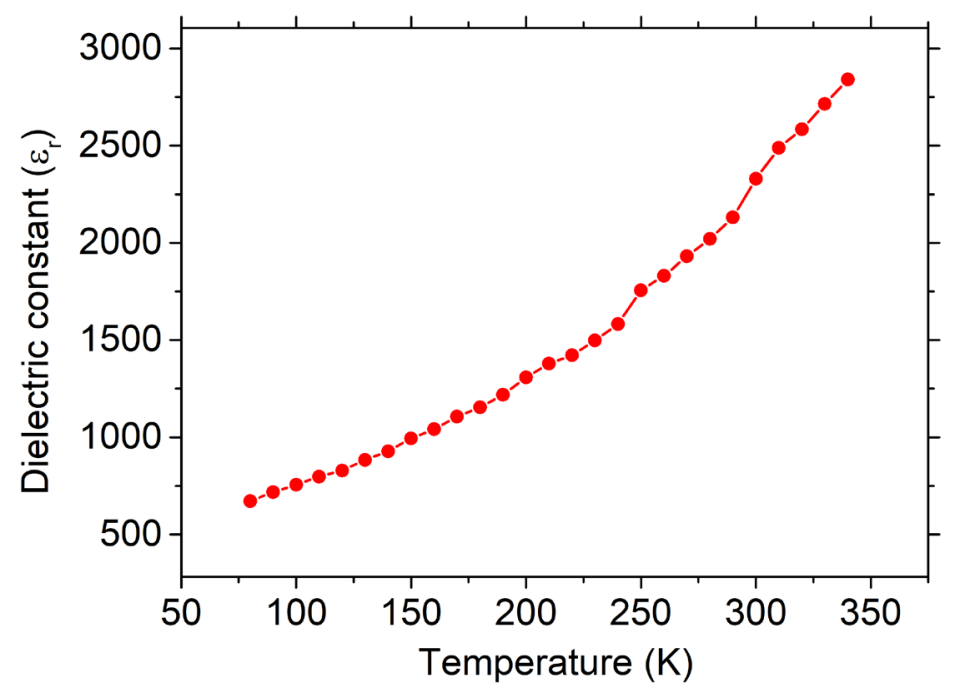

Figure 13: Dielectric permittivity obtained at $20 \mathrm{kHz}$ for the small device in the $100-320 \mathrm{~K}$ range.

When carefully comparing the results obtained for the SD to those found in the literature, one can see a good match to those presented by Zhang et al. (45) characterizing $\mathrm{Sr}^{2+}$-substituted $\boldsymbol{X P Z N}-\mathbf{0 . 1 P N} \boldsymbol{N}-(\mathbf{0 . 9}-\boldsymbol{x}) \boldsymbol{P Z T}$ ceramics. Our P-E hysteresis loop is very similar to the ones they present in the range $\boldsymbol{x}=\mathbf{0 . 2 , 0 . 3}$. Moreover, the SD 
activation energy value of $\mathbf{1 6} \mathbf{~ m e V}$ is very similar to those they obtained for $\mathrm{Sr}^{2+}$-doped 0.1PZN - 0.1PNN-0.8PZT. Our dielectric constant measurements also approximate very well to those they obtained for $0.2 P Z N-0.1 P N N-0.8 P Z T$. This is further supported by the dielectric constant obtained by Sutjarittangtham et al. (46) for $\operatorname{Sr}(6 \mathrm{~mol} \%)$ -doped 0. 2PNN - 0.8PZT ceramics at room temperature, which resembles our data at $300-320 \mathrm{~K}$.

\section{Conclusions}

In this work we have carried out an in-depth experimental study of piezoelectric PZT capacitors focused on low temperatures, where previous research is scarce. The electric characterization showed that this material exhibits excellent ferroelectric behavior at high voltages. Data extracted from the temperature measurements led us to realize that PZT became more difficult to polarize as the temperature decreased, requiring more voltage and presenting lower polarization currents. This means that reducing the temperature delays the activation of the inverse piezoelectric effect and degrades the ferroelectric properties. This behavior is confirmed by measuring the hysteresis loops at several temperatures, which reveals that coercive fields grow as temperature decreases. Polarization backswitching shows Arrhenius-like behavior in all the temperature range, being more pronounced at higher temperatures. These temperature-related effects can be explained by the fact that polarization switching is driven by domain wall motion. This is a thermally activated process, and so domain walls have less mobility or are even unable to be reversed when the temperature diminishes.

We have found that the device area influences both the poling process and coercive field. These results indicate that thinner devices show polarization domain walls with less mobility. This is mainly because thickness affects grain size. The thicker diaphragm presents larger grains, and thus, greater $180^{\circ}$ and non- $180^{\circ}$ domain wall mobility, which ease its domain reversal, explaining its faster poling process, as well as its smaller coercive field values. This hypothesis is supported by the polarization backswitching analysis, which allowed us to determine a higher activation energy of domain switching for the smaller device. Furthermore, both devices present low activation energy values, typical of a relaxor ferroelectric.

Impedance measurements of the smaller device show a global impedance maximum at $8.3 \mathrm{kHz}$, and local maxima at $6.4 \mathrm{kHz}, 11.6 \mathrm{kHz}, 15.3 \mathrm{kHz}$ and $17 \mathrm{kHz}$. These results were confirmed by electroacoustic measurements. The simultaneous frequency and temperature sweeps indicated that impedance maxima increase and shift towards higher frequencies as temperature decreases. Hence, decreasing the temperature produces an internal stress in the ceramic generating larger stiffness and, consequently, reducing its piezoelectric coefficients.

The values measured for the dielectric constant of the smaller sample, as well as its activation energy and polarization hysteresis loop are in great accordance with those found in the literature for $\mathrm{Sr}$-doped PNN-PZT based ceramics.

\section{Acknowledgments}

This work was supported by the Spanish Ministry of Science, Innovation and Universities grant TEC2017-84321-C4-2-R, with support of Feder funds. 


\section{References}

1. W. Wersing, Ferroelectric Devices, in Ferroelectric Ceramics, N. Setter and E.L Colla., Editors, Monte Veritá, Proceedings of the Centro Stefano Franscini, Ascona, Birkhäuser Basel (1993).

2. J. Krautkrämer and H. Krautkrämer, Ultrasonic Testing of Materials, pp. 119-149, Springer-Verlag, Cologne (1983).

3. H. Hoshyarmanes and A. Abbasi. Journal of Intelligent Material Systems and Structures, 29(9), pp. 1799-1817 (2018).

4. H. Hoshyarmanes, N. Ebrahimi, A. Jafari, P. Hoshyarmanesh, M. Kim, and H. Park. Sensors, 19, 13 (2019).

5. H. Mei, M. F. Haider, R. Joseph, A. Migot, and V. Giurgiutiu, Sensors, 19, 383, (2019).

6. E. Silva de Freitas, F. G. Baptista, D. E. Budoya, and B. A. de Castro, IEEE Sensors Journal, 17, pp 5537-5546 (2017).

7. E. Silva de Freitas and F. G. Baptista, Sensors and Actuators A, 238, pp. 220-228 (2016).

8. Y. Zhang, Z. Chen, W. Cao, and Z. Zhang, App. Phys. Lett., 111, 172902 (2017).

9. L. Qiao, G. Li, H. Tao, J. Wu, Z. Xu, and F. Li, Ceramics International, 46, 5, pp. 5641-5644 (2020).

10. L. Wu, D. Xiao, J. Wu, Y. Sun, D. Lin, J. Zhu, P. Yu, Y. Zhuang, and Q. Wei, Journal of the European Ceramic Society 28, pp. 2963-2968 (2008).

11. S. Singh, B. Singh, S. Kumar, and A. K. Yadav, Ferroelectrics, 551, 1, pp. $133-$ 142 (2019).

12. G. A. Samara, Ferroelectrics, 2, 277-289 (1971).

13. N. Setter, D. Damjanovic, L. Eng, G. Fox, and S. Gevorgian, J. App. Phys., 100, 051606 (2006).

14. L. W. Martin, Y.H. Chu, and R. Ramesh, Mater. Sci. Eng. R., 68, pp. 89-133 (2010).

15. S. Meena, S. M. Sze, U. Chand, and T.-Y. Tseng, Nanoscale Research Letters, 9, 526 (2014).

16. O. Auciello, J. F. Scott, and R. Ramesh, Physics Today 51(7), 22 (1998).

17. S. Hofmann-Eifert and T. Watanabem, Chapter 6: FeRAM, Atomic Layer Deposition for Semiconductors, pp. 149-171 (2014).

18. L. Wang, C. Yang, and J. Wen, Electron. Mater. Lett., 11, pp. 505-543 (2015).

19. A. Chen, Solid-State Electronics, 125, 2, pp. 5-38 (2016).

20. M. Dawber, K. M. Rabe, and J. F. Scott, Rev. Mod. Phys., 77, 4, pp. 1083-1130 (2005).

21. H. Ishiwara, Multifunctional Oxide Heterostructures (2012).

22. K. M. Rabe, M. Dawber, and C. Lichtensteiger, Modern Physics of Ferroelectrics: Essential Background, in Physics of Ferroelectrics, pp 1-30, Springer-Verlag, Berlin (2007).

23. B. Jaffe, W. R. Cook Jr., and H. Jaffe, Chapter 8 - Perovskite Niobates and Tantalates and Other Ferroelectric and Antiferroelectric Perovskites, in Piezoelectric Ceramics, pp. 185-212, Elsevier, Academic Press (1971).

24. J. F. Scott, C. A. Araujo, B. M. Melnick, L. D. McMillan, and R. Zuleeg, J. Appl. Phys., 70, 382 (1991). 
25. J. S. Cross, M. Fujiki, M. Tsukada, K. Matsuura, S. Otani, M. Tomotani, Y. Kataoka, Y. Kotaka, and Y. Goto, Integrated Ferroelectrics, 25, 1-4, pp. 265-273 (1999).

26. F. Xu, S. Trolier-McKinstry, W. Ren, and Baomin Xu., J. App. Phys., 89, 1336 (2001).

27. X. J. Meng, J. L. Sun, X. G. Wang, T. Lin, J. H. Ma, S. L. Guo, and J. H. Chu, Appl. Phys. Lett., 81, 4035 (2002).

28. J. Pérez de la Cruz, E. Joanni, P. M. Vilarinho, and A. L. Kholkin, Journal of Applied Physics, 108, 114106 (2010).

29. V. R. Mudinepalli, L. Feng, W. Lin, and B. S. Murty, Journal of Advanced Ceramics, 4, 1, pp. 46-53 (2015).

30. Y. Zhang, X. L. Zhong, Z. H. Chen, J. B. Wang, and Y. C. Zhou, Journal of Applied Physics, 110, 014102 (2011).

31. W. J. Merz, Phys. Rev., 95, 690 (1954).

32. D. Berlincourt and H. H. A. Krueger, Journal of Applied Physics, 30, 1804 (1959).

33. Dragan Damjanovic, Rep. Prog. Phys., 61, 1267 (1998).

34. Rolf Landauer, Journal of Applied Physics, 28, 227 (1957).

35. L. Tian, David A. Scrymgeour, and Venkatraman Gopalan, Journal of Applied Physics, 97, 114111 (2005).

36. B. Wen, Y. Zhang, X. Liu, L. Ma, and X. Wang, J. Mater. Sci., 47, 4299\{4304 (2012).

37. F. Chu, I. M. Reaney, and N. Setter, Journal of Applied Physics, 77, 1671 (1995).

38. G. Park, K. Kabeya, H. H. Cudney, and D. J. Inman, JSME International Journal, series $A$, Vol. 42, No. 2 (1999).

39. C. Liang, F.P. Sun, and C.A. Rogers, J. Intell. Mater. Syst. Struct., 5, pp. 12-20 (1994).

40. D. Damjanovic, Phys. Rev. B, 55, R649(R) (1997).

41. D. Damjanovic, J. App. Phys., 82, 1788 (1997).

42. Q. M. Zhang, H. Wang, N. Kim, and L. E. Cross., J. App. Phys., 75, 454 (1994).

43. Y. Y. Lim and C. K. Soh., Journal of Intelligent Material Systems and Structures, 23, 7 (2012).

44. R. G. Sabat, B. K. Mukherjee, W. Ren, and G. Yang, Journal of App. Phys., 101, $064111(2007)$.

45. Y. Zhang, X. Zhu, J. Zhu, X. Zeng, X. Feng, and J. Liao, Ceramics International, 42, pp. 4080-4089 (2016).

46. K. Sutjarittangtham, N. Tawichai, U. Intatha, S. Eitssayeam, K. Pengpat, and G. Rujijanagul, Ferroelectrics, 384, 1, pp. 56-61 (2009). 